\title{
The PLExus Prototype: A PLE realized as Topic Maps
}

\author{
Kolås, L. and Staupe, A. \\ Department of Computer and Information Science, Norwegian University of Science and \\ Technology, Sem Scelandsveg 7-9, 7491 Trondheim, Norway \\ linek@idi.ntnu.no,ahs@idi.ntnu.no
}

\begin{abstract}
The article presents the PLExus prototype, a Personal Learning Environment based on the semantic technology of topic maps. Semantic-based navigation in e-learning will enable variation, differentiation and individualization, which are important pedagogical factors in the development of a personal learning environment. PLExus provides a student interface allowing customized views of learning objects and learning activities based on pedagogical method, media type, learning objective type, proficiency stage etc.
\end{abstract}

\section{Introduction}

An HCI solution offering a customized interface within e-learning could be realized using the semantic technology of topic maps, where the information can be shown in several views based on the choice of the user. Topic maps are an ISO standard - ISO/IEC 13250:2003. "A topic map is a technology for knowledge integration, describing concepts and their relations" [1]. Organizing documents into a topic map, it is necessary to identify the topics, the topic types, the occurrences and the associations [2].

This article first describes topic maps within elearning, where topic maps may be a solution to achieve a personalized learning environment. A description of important primary constructions in topic maps applicable in a PLE is presented. Finally PLExus, the PLE prototype is presented with screenshots and a system description.

Based on the development of the functional requirements [3], one of the experiences was that a next-generation e-learning system must be a personal learning environment (PLE). Johnson et al. describes how individuals have different understandings of the concept "PLE", from "empowering users of informal learning resources away from institutions" or "an extended portfolio" to "a superfluous accessory to the technologies of the desktop operating systems and the World Wide Web" [4]. The variety of interpretation illustrates how diffuse the concept of PLE still is. This article will present our interpretation of the concept "PLE" based on the experiences developing pedagogical-based topic maps.

\section{An e-learning topic map}

In a pedagogical-based PLE like PLExus the student is able to customize the learning environment. This requires that Learning objects (LO) and learning activities (LA) are saved and retrieved in such a manner that one student could reach the learning objective through a presentation, while other students reach the same learning objective through e.g. discovery, demonstration or collaboration [5].

The student interface based on a topic map allows customized views of the LO and LA. The students' views will be based on e.g: themes, time (the newest LO / LA), pedagogical methods [5], media type / intelligence [6], proficiency stages [7], learning objective (knowledge/skill/attitude), student productions of LO/LA, ranking score (LO with the highest scores), list of LO recommended by the system based on behavior of previous students, guided learning paths produced by teacher and free text search.

\section{Topic Maps in E-learning and Personal Learning Environments}

A user-friendly, individualized and differentiated interface is an important feature of an e-learning system. Instead of presenting the LO/LA in one 
standard interface for all the students, an e-learning topic map presents "many roads to Rome", addressing the needs of the heterogeneous student group [8]. Dichev et al. [9] mention many advantages using topic maps, e.g. efficient context-based retrieval, customized views, information visualizations and deeper understanding of the domain conceptual relations. The advantages of a topic map presenting information (e.g. LO) are that the user will experience a flexible learning environment and is able to make his / her choices on what perspective s/he wants to the learning material.

Information overload for the student is a problem which may occur when we are trying to arrange for an individualized and differentiated learning environment prepared for individual needs when it comes to methods, media, intellectual stages, cultural needs, assessment and different intelligences [3] in the existing online learning environments. If nothing else is done other than organizing many different LOs into folders, the students will not know which LO to start with and which to continue with. With a chronological structure of the folders (week 1, week 2...) it will be hard for the students to relocate e.g. the World War 2lesson. A folder structure based on themes makes it hard to individualize / differentiate the lessons e.g. by using a variety of pedagogical methods (the student then will have to choose from WW2-demo, WW2simulation, WW2-discussion etc). Adding other pedagogical factors e.g. several proficiency stages will make navigation in the online learning environment even harder.

In order to meet the requirements of a PLE, a powerful computer architecture is needed, where it is easy to locate resources based on context and needs. There should also be a powerful search- and navigation system connected to the architecture. The architecture must ensure relevant, complete and consistent information. One example of this type of architecture is Topic Maps.

For a PLE we need a system for administering a certain amount of information which is in constant change, normally growing, and which also consists of a variety of information that can be linked together in many different ways. That means administration of complex information. In Topic Maps, metadata can be isolated and stored separately from the object, but will still be closely connected to the object. Metadata will be a central component during information search.

\section{Realizing a PLE using Topic Maps}

In this article we focus more on the strength of topic maps in order to create a PLE, and therefore, to some extent, we describe the qualities of parts of the primary structure in topic maps. Previously we have described the use of LOs [3]. The LOs may exist many places, in a local database, in a publisher's database, available on the Internet, etc. In the first place, LOs can be made of images, text files, animations, videos, etc. without necessarily being used / viewed as LOs. To stick to the terminology of topic maps we therefore call these "subjects".

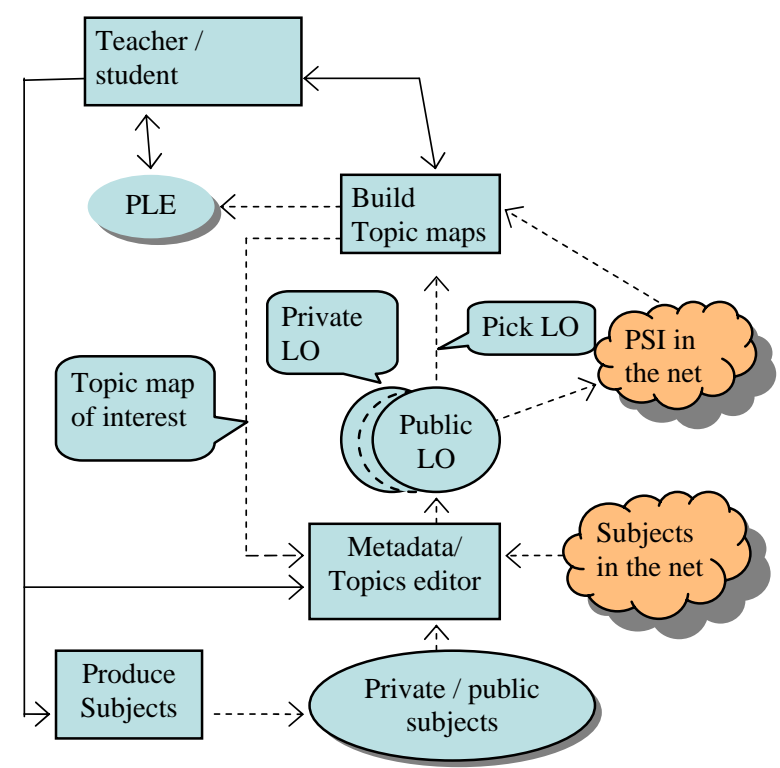

Figure 1: The conceptual model

The metadata connected to these subjects will only be those connected to ordinary files. Based on the subjects, it should be possible to create LOs containing additional metadata necessary when the object is to be used in learning situations. In turn, one might want to create new LOs around one, two, or several basic LOs. The new LO will then get its metadata and can be made available. And we could go on like this; several LOs forming parts of courses, course modules, or sometimes a complete course in a PLE.

The subjects can originate from many different sources, e.g. images, text files, web pages, videos etc. Not all subjects can be put into a computer or be directly connected to it, the alternative will then be to describe it in the form of a substitute (proxy) in the topic map architecture. 
The conceptual model (fig. 1) is built around the use of topic maps, since we believe that topic maps are suitable as the core of a powerful PLE with information administration, search and navigation as important components. Topic maps are "the GPS of the information universe" [10], it tells us where we are and where to find the requested information.

\subsection{Teacher}

The model is based on the assumption that both teachers and students can act as consumers and producers in an online learning environment. A typical situation is when a teacher is creating a course or a course module. The teacher will then check if any LOs already exist. S/he searches and reuses LOs from his/her own library, locally published LOs or from an internet-based PSI (explained in 6.1).

If no suitable or preferred LO exists, the teacher can choose to develop his/her own. S/he may use already-existing subjects in the form of images, text, animations, videos, evaluation programs, arenas of cooperation, toolkits etc. In the process of transforming subjects into LOs (topics), it will be necessary to add metadata and PSI (see 6.1). The PLExus editor builds the topics with the necessary elements (base names, possible variant names, occurrence(s), scope(s) and subject indicator). The LO is added to a private or a public database, or in both.

The next step will be to build a topic map with the topics, association hierarchies and class hierarchies. When the final step of the topic map construction is completed, one will have a complete PLE. However, the road there could include several steps where topic maps are expanded. Each of these steps may lead to topic maps that are interesting enough to be made public, or to be added to a private database of LOs.

\subsection{Student}

Students will get access to a learning environment as both producers and consumers of LO/LA. The most extreme case is that students build the content of the entire PLE. A more common example is that the students design their own electronic workbook, develop smaller course modules etc. As for the teacher, it is natural that the students work in relation to private LO bases and it will be necessary with authorization before the $\mathrm{LO}$ is made publicly available.

\section{PLExus - a prototype of a pedagogical- based PLE}

We here introduce PLExus - a prototype of a pedagogical-based PLE realized as a topic map. The student experiences a personalized user interface where s/he gets access to the LOs from different points of view, e.g. pedagogical method (e.g. game, tutorial, discussion etc), proficiency stage (e.g. novice, competence etc), or intelligence (visual, verbal etc). The PLExus prototype presented is at this point in time only covering a few views, but the basic structure is implemented. PLExus is based on our experiences with LOs and writing metadata with design patterns [3].

\subsection{The wizard}

The teacher prepares a personal learning environment for the student by structuring the LOs using a pedagogical topic map wizard. The prototype is operational, but a self-instructing user interface is not yet implemented. We first present the wizard where the teacher adds a new LO to the system, then presents the student view of the system.

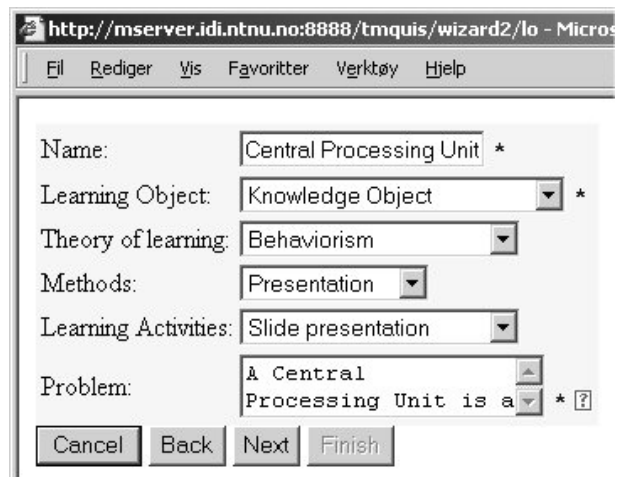

Figure 2: The wizard - Adding initial metadata.

In fig. 2 the teacher fills in metadata, partly by choosing from a list, partly by writing free text. In the existing version of the prototype, the metadata covered in this phase include: name, LO type, theory of learning, pedagogical method, type of learning activity and problem description. The question marks provide help to the user.

The next step is to add the learning part by providing a URL. We use design patterns to describe the learning parts, by introducing the idea of creating the metadata in several steps. The making of an LO with metadata will then be one process, instead of two 
separate parts where one first create the learning part and then create the metadata.

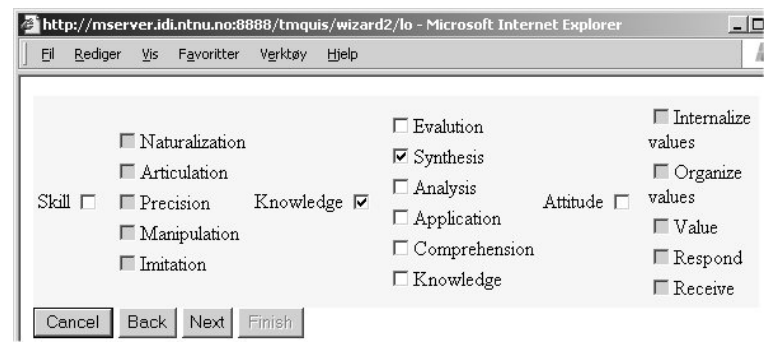

Figure 4: The wizard -defining learning objective type and taxonomy level.

The teacher must mark what learning objective type and taxonomy level(s) the LO covers (fig. 4). In fig. 5 the teacher fills in metadata covering solution, steering/ control, participation, construction, limitations and example usage [3]. After adding the metadata the teacher saves the LO (with metadata) to the topic map.

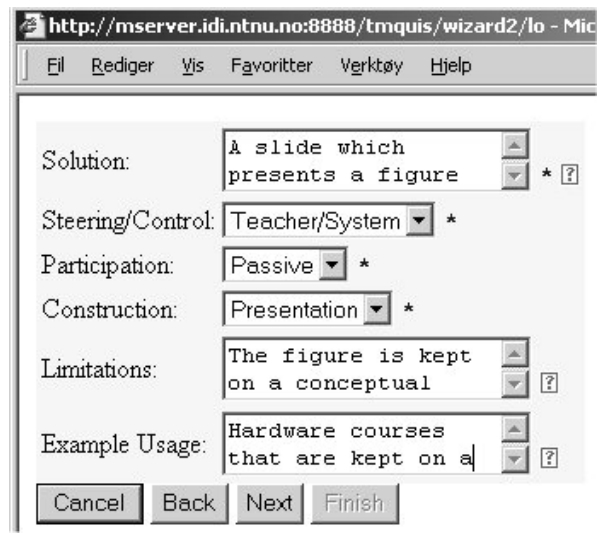

Figure 5: The wizard - Adding metadata.

Fig. 6 illustrates how the student currently experiences the learning environment; the chosen LO, which is of type "knowledge object" is shown on the left side of the screen. All the links on the right side of the screen enable semantic-based navigation between LOs. Currently, the prototype provides only a few navigation opportunities (based on theory of learning and ROOs), but the student will in the future have access to LOs based on e.g. pedagogical method, theme, media type, ranking score etc.

The second block on the right side of the screen shows that the knowledge object "Central Processing Unit” is part of the resource organizing object (ROO) "CPU". It also shows the other LOs in the current $\mathrm{ROO}$, in this case the knowledge object (KO) "Control
Unit”. Its purpose is to enable navigation between objects in the resource organizing object. The third block shows and provides access to all the ROO the KO "Central Processing Unit" is part of. The links enable the user to change context without leaving the current knowledge object.



Figure 6: Example of topic map screenshot.

\section{System overview}

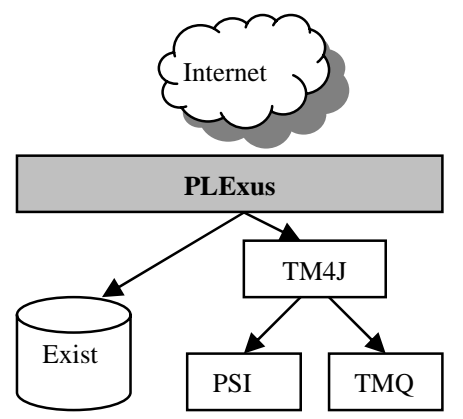

Figure 7: System overview

Explanation of fig. 7:

- "Exist” [11] is an XML database, which stores raw data without knowledge of metadata.

- TM4J [12]: The Topic Map engine, integrated through TM4Web [13].

- PSI: The Published Subject Identifier in topic maps.

- TMQ: The QUIS topic map with LOs and metadata.

\subsection{The PSI Topic Map}

A PSI (Published Subject Identifier) is necessary to ensure that the same topics are assigned the same topic names and should in the future be standardized by the educational field. An educational PSI must be made 
available via Internet. Such environments already exist within several subject areas. The PSI topic map is in our work of the PLExus prototype a local and temporal variant of a PSI.

\subsection{The TMQ Topic Map}



Figure 8: A KO with a variety of associations.

TMQ (topic map QUIS) in fig. 7 is the topic map prototype containing the metadata for the subjects / raw data. Each LO has metadata directly connected to itself as resourceData in occurrences. The metadata (resourceData) is written and searchable in free text. The metadata will in the topic map-based user interface provide semantic navigation. There are five LO types; knowledge, monitor, test, tool and resource organizing object [3]. The first four of these will have similar constructions. The resource organizing object will in addition to similar associations also have a wrapper.

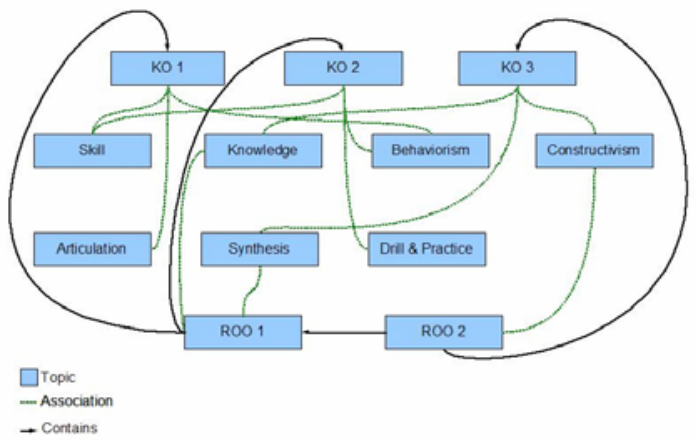

Figure 9: Example: The content of $\mathrm{ROO} 2$ is $\mathrm{KO} 3$ and ROO1 (existing of $\mathrm{KO} 1$ and $\mathrm{KO} 2$ ).

A Knowledge Object (KO) topic is an LO topic, pointing to the subject. It also has an association binding the topic to a common $\mathrm{KO}$ topic, identifying it as a $\mathrm{KO}$ and allowing easier access by queries. The second LO type implemented in the prototype is the resource organizing object (ROO). As the $\mathrm{KO}$, the $\mathrm{ROO}$ is an LO associated to identifying topic. The ROO's function is to bind together several LOs, which could be other ROOs or KOs (fig. 9).

\section{Conclusions}

The article has presented PLExus, which is a prototype of a PLE realized with the topic map technology. The term "plexus" means "network", which very well describes a PLE based on the semantic technology of topic maps. Semantic-based navigation in e-learning will enable variation, differentiation and individualization, which are important factors developing a personal learning environment.

\section{References}

[1] L.M. Garshol, Topic maps - overview and use cases. http://www.vestforsk.no/seminar/Ontopia.pdf, 2006.

[2] S. Pepper. The TAO of Topic maps - Finding the way in the age of infoglut. http://www.ontopia.net/topicmaps/materials/tao.html, 2002. [3] L. Kolås, A. Staupe, Requirement specification for a next generation e-learning system. http://www2.tisip.no/quis/public_files.php, 2006.

[4] M.Johnson, O.Liber, S.Wilson, P.Sharples, C.Milligan, Beauvoir, P. Mapping the future: The personal learning environment reference model and emerging technology. ALT-C 2006 The next generation Research proceedings. 2006, ISBN 0-9545870-5-7

[5] R., Heinich, M., Molenda, J.D. Russell, and Smaldino, S.E. Instructional media and technologies for learning 7th edition. (Merrill Prentice Hall. 2002).

[6] H. Gardner, Frames of Mind: The Theory of Multiple Intelligences. (New York; Basic Books. 1985).

[7] H.L. Dreyfus, Intelligence without representation, http://www.hfac.uh.edu/cogsci/dreyfus.html, 1998.

[8] L, Kolås, Variation and Reusability in E-learning: not Compatible? E-learn 2005 Proceedings. AACE.

[9] C. Dichev, D. Dichva, L. Aroyo, Using topic maps for elearning. In proceedings of the IASTED International Conference CATE, 2003.

[10] C. F. Goldfarb, White paper - The topic maps handbook, Empolis, http://www.empolis.com/downloads/ empolis_TopicMaps_Whitepaper20030206.pdf, 2003

[11] Exist, eXist - Open source native XML database. http://exist.sourceforge.net, (2006).

[12] TM4J, Topic maps for java. http://tm4j.org, 2006. 
[13] TM4Web, TM4Web - Topic maps for web.

http://tm4j.org/tm4web.html, 2006. 